\title{
Methodological utility of chemerin as a novel biomarker of immunity and metabolism
}

\author{
Fabian Eichelmann', Cornelia Weikert ${ }^{2,3}$, Romina di Giuseppe ${ }^{4}$, Ronald Biemann ${ }^{5}$, \\ Berend Isermann ${ }^{5}$, Matthias B Schulze ${ }^{6,7}$, Heiner Boeing ${ }^{8}$ and \\ Krasimira Aleksandrova ${ }^{1}$
}

'Department of Epidemiology, Nutrition, Immunity and Metabolism Start-up Lab, German Institute of Human Nutrition Potsdam-Rehbruecke (DIfE), Nuthetal, Germany

${ }^{2}$ Department of Food Safety, Federal Institute for Risk Assessment, Berlin, Germany

${ }^{3}$ Institute for Social Medicine, Epidemiology and Health Economics, Charité University Medical Center, Berlin, Germany

${ }^{4}$ Institute of Epidemiology, Christian-Albrechts University Kiel, Kiel, Germany

${ }^{5}$ Department for Clinical Chemistry and Pathobiochemistry, Otto-von-Guericke-University Magdeburg, Magdeburg, Germany

${ }^{6}$ Department of Molecular Epidemiology, German Institute of Human Nutrition Potsdam-Rehbruecke (DIfE), Nuthetal, Germany

${ }^{7}$ German Center of Diabetes Research (DZD), München-Neuherberg, Germany

${ }^{8}$ Department of Epidemiology, German Institute of Human Nutrition Potsdam-Rehbruecke (DIfE),

Nuthetal, Germany

Correspondence

should be addressed

to K Aleksandrova

Email

Krasimira.Aleksandrova@

dife.de

\begin{abstract}
Chemerin is a recently discovered adipokine with inflammatory and metabolic actions relevant for chronic disease development. However, evidence from human research on the role of chemerin in chronic disease risk is still lacking. We assessed the reliability of plasma chemerin concentrations measured on two occasions over a 4-month period in 207 apparently healthy participants. In addition, we explored the cross-sectional associations between chemerin and inflammatory biomarkers using Spearman partial correlation and multivariable linear regression analyses. Intra-individual reproducibility of chemerin measurements was assessed by calculating intraclass correlation coefficients (ICCS) and exploration of Bland-Altman plots. Reliability analyses revealed good reproducibility of chemerin measurements (ICC: $0.72(95 \%-\mathrm{Cl} 0.65,0.78)$ ). Visual inspection of Bland-Altman plots confirmed that the two time point measurements had a high level of agreement. In correlation analyses, chemerin was positively correlated with adiposity measures (body mass index and waist circumference). In addition, independent of adiposity measures, chemerin was correlated with the biomarkers C-reactive protein, fatty acid-binding protein 4 and progranulin (Rho-s ranging from 0.23 to 0.37 ). In multivariable linear regression analysis, a combination of correlated factors including body mass index, waist circumference, C-reactive protein, progranulin and fatty acid-binding protein- 4 explained $28.0 \%$ of chemerin concentrations. These findings demonstrate methodological utility of chemerin concentrations in populationbased research setting. Human studies are highly warranted in order to provide further insights into the role of chemerin as a biomarker linking immunity and metabolism in relation to chronic disease risk.
\end{abstract}

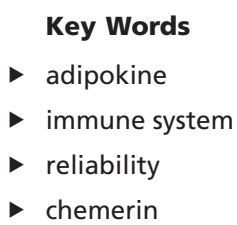

Endocrine Connections (2017) 6, 340-347

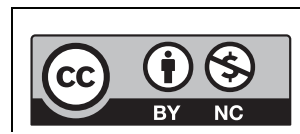

This work is licensed under a Creative Commons Attribution-NonCommercial 4.0 International License. http://www.endocrineconnections.org DOI: 10.1530/EC-17-0098 (c) 2017 The authors Published by Bioscientifica Ltd 


\section{Introduction}

Chemerin, also known as tazarotene-induced gene 2 protein (TIG2) and retinoic acid receptor responder 2 (RARRES2), is a $14-\mathrm{kDa}$ chemoattractant protein known to modulate immune system function through its binding to the chemerin receptor ChemR23 (1). It is secreted in an inactive form as prochemerin and is activated through cleavage of the C-terminus by inflammatory and coagulation serine proteases. Animal studies indicated visceral adipose tissue, placenta and liver, and to a lesser extent lungs, heart, ovaries, kidneys and pancreas as main anatomical sites of chemerin production $(2,3)$. Chemerin was initially identified as a retinoid-responsive gene present in psoriatic skin lesions in 1997 using differential display (4). Ten years later (in 2007) chemerin was re-discovered as a novel adipokine regulating adipogenesis and genes critical in glucose and lipid metabolism of adipocytes (3). Subsequent research characterized additional roles of chemerin in diverse biological processes including cell proliferation and differentiation, angiogenesis, renal function and energy metabolism (3, 5, 6, 7). Collectively, chemerin could be distinguished as an immune marker acting as a chemoattractant agent promoting the recruitment of immune cells to lymphoid organs and sites of tissue damage and as autocrine/paracrine signaling agent that is involved in several metabolic and celldevelopmental processes particularly in adipocytes (8). Higher circulating chemerin concentrations have been reported in patients suffering from systemic inflammatory diseases such as Crohn's disease, ulcerative colitis (9), liver diseases (10), chronic pancreatitis (11) and polycystic ovary syndrome (12). Cross-sectional studies reported associations between chemerin and biomarkers of inflammation, metabolic parameters and anthropometric measures of adiposity (13). However, little is known about the interrelations of chemerin with inflammation-related adipokines. Particularly, there have been no studies based on human population samples.

In summary, recent research has uncovered chemerin as an adipokine implicated in immune, inflammatory and obesity-related pathophysiological conditions. However, no data from large-scale prospective population-based studies exist to support or refute generated hypothesis on chemerin involvement in disease development. Important prerequisite for planning biomarker assessment in a prospective study is the assessment of whether single biomarker measurements, as done in most epidemiological research settings, are stable over time and not affected by biological variability (14). So far, the reliability of chemerin as biomarker in human research has not been evaluated. To fill this gap, we aimed to assess the intra-individual reproducibility of plasma chemerin concentrations over a 4-month interval using data from a sample of apparently healthy participants within the European Prospective Investigation into Cancer and Nutrition (EPIC) Potsdam Study. As a secondary aim, we analyzed the cross-sectional association of chemerin with inflammation-related adipokines.

\section{Methods}

\section{Study population}

The EPIC-Potsdam Study is part of the multicenter prospective cohort study EPIC, which was designed to investigate the association between nutrition, cancer and other chronic diseases (15). In Potsdam, Germany, 27,548 participants (16,644 women aged 35-65 years and 10,904 men aged 40-64) were recruited between 1994 and 1998 (16). The present study was based on a randomly selected sample from all participants of the EPIC-Potsdam Study population (17). Exclusion criteria included history of heart disease and stroke (myocardial infarction, heart failure, cardiomyopathy and angina pectoris), impaired mobility, current therapy with $\beta$-blockers or systolic or diastolic blood pressure above $180 \mathrm{mmHg}$ or $110 \mathrm{mmHg}$, respectively. Of the initially invited 407 participants, 11 did not respond to the invitation, 176 declined study participation. Twelve participants were additionally excluded due to reported therapy of $\beta$-blockers. From the remaining participants, all but one provided blood samples leading to a total sample size of 207 participants (124 women and 83 men). Blood samples were collected on two occasions 4 months apart with the first in the period of October 2007-March 2008 and the second between February and July 2008. Timing of blood collection was, with few exceptions, 08:00-11:00h, with ca. 10\% of participants not being fasted. All participants provided written informed consent and the study procedures were approved by the Ethics Committee of the Medical Association of the State of Brandenburg.

\section{Biomarker measurements}

On two occasions, four months apart, blood was drawn, the fractions separated and stored at $-80^{\circ} \mathrm{C}$. With the exception

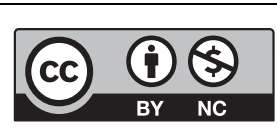

This work is licensed under a Creative Commons Attribution-NonCommercial 4.0 International License. 
of high-sensitivity C-reactive protein (hsCRP), which was measured in serum, concentrations of chemerin, fatty acidbinding protein 4 (FABP-4), monocyte chemoattractant protein 1 (MCP-1), omentin-1, progranulin and vaspin were measured in EDTA-plasma. All biomarkers were measured with commercially available sandwich enzymelinked immunosorbent assays (ELISA) (BioVendor, Kassel, Germany) according to the manufacturer's instructions. Repeated samples of each participant were measured in the same analytical batch. Measurements of chemerin and omentin-1 were performed at the Institute of Clinical Chemistry, University Magdeburg. Manufacturer's specified average intra- and inter-assay coefficients of variation $(\mathrm{CV})$ for chemerin were $6.0 \%$ and $7.6 \%$, respectively. Inter-assay $\mathrm{CV}$ based on our data was $5.9 \%$. Measurements of hsCRP in serum and FABP-4, MCP-1, progranulin and vaspin in plasma were performed in the Department of Clinical Nutrition, German Institute of Human Nutrition Potsdam-Rehbrücke, Germany. The intra- and inter-individual CVs of hsCRP were 5.1\% and $6.1 \%$, respectively as reported by the manufacturer. Based on our data, the inter-individual CV was $11.0 \%$. Due to insufficient serum volume, hsCRP could only be measured in a reduced number of participants $(n=151)$. Detailed information on the measurements of plasma FABP-4, MCP-1, omentin-1, progranulin and vaspin concentrations have been reported elsewhere $(18,19)$.

\section{Statistics}

Non-normally distributed data were transformed using the natural logarithm in order to allow the application of parametric tests and the calculation of mean concentrations. Non-normality was determined by visual inspection of the histogram and quantile-quantile plot evaluation. Mean chemerin concentrations are presented as geometric means. Strata-specific mean concentrations were calculated for sex, BMI $\left(<25 \mathrm{~kg} / \mathrm{m}^{2}\right.$ vs $\left.\geq 25 \mathrm{~kg} / \mathrm{m}^{2}\right)$, waist circumference ( 0 : $<94 \mathrm{~cm}$ vs $\geq 94 \mathrm{~cm}$, $\circ:<80 \mathrm{~cm}$ vs $\geq 80 \mathrm{~cm}$ ) and inflammatory status represented by hsCRP concentration (below sample median vs above median). A random-effects analysis of variance (ANOVA) with chemerin concentration as dependent variable and study participant as explanatory variable was used to estimate the variance components explained by within-person and between-person differences, respectively. Overall and strata-specific intraclass correlation coefficients (ICCs) were calculated as ratios of between-person variance and total variance (betweenperson variance + within-person variance).

To compare mean chemerin concentrations between sampling time points and between categories within one sampling time point, paired and unpaired twosided Student's $t$-tests were applied. In addition to ICCs, reliability was also evaluated by inspection of Bland-Altman plots as a recommended complementing procedure to assess the agreement of two measurements for each participant (20). To evaluate interdependence with the individual characteristics, the ICCs were calculated across the above defined strata (sex, BMI, waist circumference and hsCRP levels). Following established cut points, estimated reproducibility is rated as excellent (ICCs $\geq 0.75$ ), good (ICC: 0.74-0.60), fair (ICC: 0.59-0.40) or poor (ICCs $<0.40$ ). In sensitivity analyses, ICCs were calculated after excluding participants with extreme chemerin measurement values (values within 1st or above 99th percentile) and non-fasting participants at blood sampling. Using linear regression, we examined the influence of time of day at blood draw taking into account age, sex, fasting status at blood draw and BMI.

Correlation of chemerin with anthropometric measures of adiposity (BMI and waist circumference) and selected biomarkers, including hsCRP, FABP-4, MCP-1, omentin-1, progranulin and vaspin were evaluated using Spearman partial correlation analyses adjusted for age and sex. Correlation analyses with the biomarkers were additionally adjusted for BMI. Fisher's $z$ transformation was used to produce $95 \%$ confidence intervals for each correlation coefficient. In linear regression analyses, we modeled anthropometric measures and biomarkers as predictors of chemerin concentrations to estimate the explained variance represented by the adjusted coefficient of determination (adjusted $R^{2}$ ). Adjusted $R^{2}$ takes into account the number of predictors in the model, which automatically would increase the explained variance with every additionally included variable without actually having a predictive value. In correlation and regression analyses anthropometric indices and biomarker concentrations of the first (baseline) measurement were used as these did not substantially differ from the second measurement. We also calculated the degree of attenuation of hypothetical relative risk estimates (RR) that could arise due to biological variability of chemerin based on the following formula: $\mathrm{RR}_{\text {true }}=\mathrm{e}^{\left(\ln \left(\mathrm{RR}_{\text {observed }}\right)^{*} \frac{1}{\mathrm{ICC}}\right)}$. All statistical analyses were performed in SAS (version 9.4, Enterprise Guide 6.1, SAS Institute Inc., Cary, NC, USA). http://www.endocrineconnections.org DOI: 10.1530/EC-17-0098
() 2017 The authors Published by Bioscientifica Ltd
This work is licensed under a Creative Commons Attribution-NonCommercial 4.0 International License. 
Table 1 Baseline characteristics of the study population, overall and by sex ${ }^{\mathrm{A}}$.

Age (years)
Range
BMI (kg/m²)
Range
Waist circumference $(\mathrm{cm})$
Range
Non-fasting $(\%)$
Chemerin $(\mathrm{ng} / \mathrm{mL})$
Range
hsCRP ( $\mu \mathrm{g} / \mathrm{mL})^{\mathrm{B}}$
Range
FABP-4 $(\mathrm{ng} / \mathrm{mL})$
Range
MCP-1 $(\mathrm{ng} / \mathrm{mL})$
Range
Omentin-1 $(\mathrm{ng} / \mathrm{mL})$
Range
Progranulin $(\mathrm{pg} / \mathrm{mL})$
Range
Vaspin $(\mathrm{ng} / \mathrm{mL})$
Range

\begin{tabular}{c}
\hline All participants $(n=207)$ \\
\hline $56.5(4.2)$ \\
$44.8-63.9$ \\
$26.5(4.0)$ \\
$19.1-41.7$ \\
$93.0(12.8)$ \\
$68.3-126.4$ \\
10 \\
$159(137,186)$ \\
$82.4-283$ \\
$1.24(0.65,2.45)$ \\
$0.14-13.4$ \\
$22.8(17.5,27.6)$ \\
$0.25-55.0$ \\
$0.44(0.35,0.53)$ \\
$0.15-1.62$ \\
$382(313,467)$ \\
$99.4-945$ \\
$30.8(26.35,36.14)$ \\
$0.40-61.0$ \\
$0.20(0.14,0.32)$ \\
$0.03-7.08$
\end{tabular}

\begin{tabular}{c}
\hline Men $(n=83)$ \\
\hline $58.0(3.1)$ \\
$51.5-63.7$ \\
$27.6(3.5)$ \\
$19.8-37.0$ \\
$101.7(10.3)$ \\
$79.3-126.4$ \\
13 \\
$162(139,186)$ \\
$82.4-283$ \\
$1.52(0.72,2.90)$ \\
$0.14-12.9$ \\
$19.7(15.1,23.4)$ \\
$8.0-54.9$ \\
$0.43(0.36,0.51)$ \\
$0.19-1.32$ \\
$362(306,433)$ \\
$99.4-945$ \\
$32.8(26.3,38.4)$ \\
$0.40-61.0$ \\
$0.18(0.12,0.22)$ \\
$0.03-5.61$
\end{tabular}

\begin{tabular}{c} 
Women $(n=124)$ \\
\hline $55.4(4.5)$ \\
$44.83-63.9$ \\
$25.8(4.1)$ \\
$19.1-41.7$ \\
$87.1(10.7)$ \\
$68.3-115.8$ \\
8 \\
$156(135,188)$ \\
$105-278$ \\
$1.10(0.58,2.21)$ \\
$0.16-13.4$ \\
$25.4(20.8,30.4)$ \\
$0.25-55.0$ \\
$0.44(0.34,0.54)$ \\
$0.15-1.62$ \\
$396(319,492)$ \\
$163-789$ \\
$30.2(26.4,34.6)$ \\
$0.45-59.5$ \\
$0.24(0.15,0.36)$ \\
$0.04-7.08$
\end{tabular}

AValues are expressed as arithmetic means (s.D.), medians (25th, 75th percentile) or percentages. ${ }^{B}$ Reduced number of participants due to insufficient serum volume.

FABP-4, fatty acid-binding protein-4; hsCRP, high-sensitivity C-reactive protein; MCP-1, monocyte chemoattractant protein 1.

\section{Results}

Table 1 presents baseline characteristics of the study population. The mean (s.D.) age of the participants was 56.5 (4.2) years. On average, participants had a BMI of $26.0(4.0) \mathrm{kg} / \mathrm{m}^{2}$ and a WC of $93.0(12.8) \mathrm{cm}$. BMI and waist circumference measurements were stable over the period of 4 months (ICC $=0.99$, ICC $=0.98$, respectively).

Table 2 presents overall and strata-specific chemerin concentrations and ICCs. Chemerin concentrations were higher in participants with higher BMI $\left(\geq 25 \mathrm{~kg} / \mathrm{m}^{2}\right)$, WC (o: $\geq 80 \mathrm{~cm}$, ơ: $\geq 94 \mathrm{~cm}$ ) and hsCRP (above $1.3 \mu \mathrm{g} / \mathrm{mL}$ )

Table 2 Overall and stratified concentrations (geometric mean (95\%-Cl)) in ng/mL for measurements taken 4 months apart and ICCs.

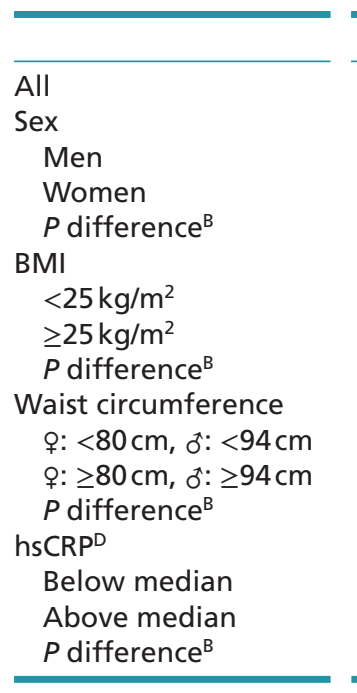

\begin{tabular}{r}
$\boldsymbol{N}$ \\
\hline 207 \\
83 \\
124
\end{tabular}

\begin{tabular}{l} 
First measurement \\
\hline $161.0(156.0,166.0)$ \\
$163.4(155.1,172.0)$ \\
$159.4(153.3,165.7)$ \\
0.45
\end{tabular}

81

126

56
151$$
70
$$

$145.8(139.3,152.7)$ $171.5(165.1,178.2)$ $<0.001$

\begin{tabular}{l}
\hline Second measurement \\
\hline $155.6(150.5,160.9)$ \\
$155.5(147.6,163.8)$ \\
$155.7(149.1,162.7)$ \\
0.97
\end{tabular}

$141.2(134.7,148.0)$
$169.0(163.0,175.2)$
$<0.001$

$149.8(143.2,156.8)$
$170.3(161.3,179.7)$
$<0.001$

$139.3(133.2,145.7)$ $167.1(160.2,174.3)$ $<0.001$

$136.5(129.6,143.9)$ $163.4(157.2,169.8)$ $<0.001$

$145.1(138.1,152.5)$

$164.3(155.3,173.9)$ $<0.001$

\begin{tabular}{ccc}
\hline \multicolumn{1}{c}{$\boldsymbol{P}$ difference ${ }^{\mathbf{A}}$} & & ICC $^{\mathbf{C}}$ \\
\cline { 1 - 1 } 0.005 & & $0.72(0.65,0.78)$ \\
0.01 & & $0.73(0.61,0.82)$ \\
0.14 & & $0.72(0.63,0.80)$ \\
& \\
0.004 & & $0.75(0.64,0.83)$ \\
0.13 & & $0.64(0.54,0.74)$ \\
& \\
0.04 & $0.78(0.65,0.86)$ \\
0.03 & $0.66(0.57,0.75)$ \\
& \\
0.04 & $0.78(0.67,0.86)$ \\
0.05 & $0.78(0.68,0.86)$ \\
&
\end{tabular}

APaired two-sided $t$-test to compare concentrations between first and second measurement. ${ }^{B}$ Unpaired two-sided $t$-test to compare concentrations between strata for sex, age, waist circumference and hsCRP levels. CICC defined as ratio of between-person variance and total variance. ${ }^{\mathrm{D}}$ Median hsCRP concentration $=1.27 \mu \mathrm{g} / \mathrm{mL}$, reduced number of participants due to insufficient serum volume.

\begin{tabular}{|lr}
\hline http://www.endocrineconnections.org & ○ 2017 The authors \\
DOI: 10.1530/EC-17-0098 & Published by Bioscientifica Ltd
\end{tabular}

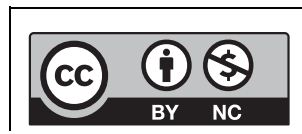

This work is licensed under a Creative Commons Attribution-NonCommercial 4.0 International License. 


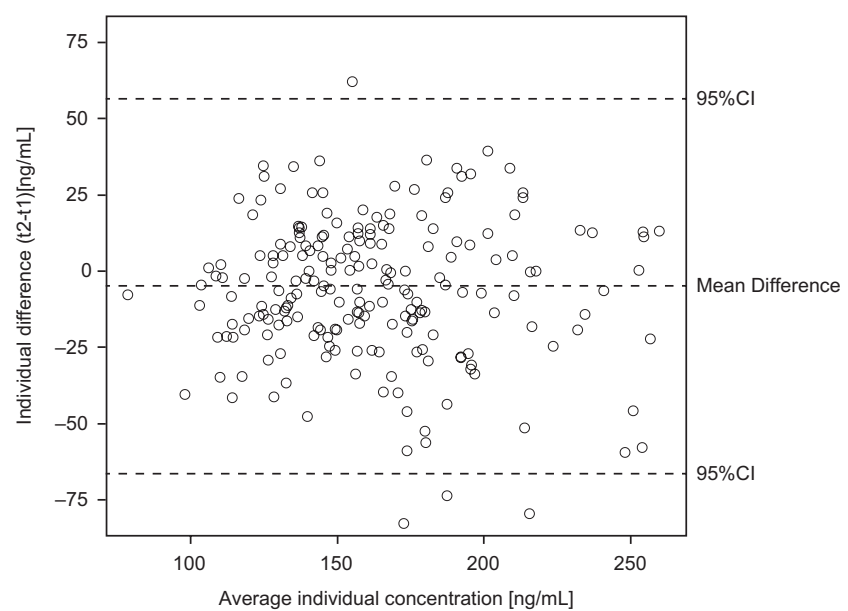

\section{Figure 1}

Bland-Altman plot. Agreement of repeated measurements ( $y$-axis) in relation to average concentrations ( $x$-axis) for each individual $(n=207)$. Agreement was calculated as difference between the two measurements (t2-t1) for each individual. 95\% confidence bounds represent the expected range of differences based on the average difference. Values of one participant were excluded from the figure, but not from calculation of mean difference and $95 \% \mathrm{Cl}$, to improve the legibility of the figure. Values for this participant were individual difference $=297.7(\mathrm{ng} / \mathrm{mL})$, average individual concentration $=258.5(\mathrm{ng} / \mathrm{mL})$.

compared to lower strata. The overall ICC of a 4-month period was 0.72 (95\%-CI: $0.65,0.78$ ), indicating 'good' reliability. Strata-specific ICCs did not substantially differ between the sexes and according to strata of BMI, WC and hsCRP levels staying within the interval of 'good' reliability (Table 2). Information from the Bland-Altman plots (Fig. 1) essentially confirmed a good agreement and symmetric distribution of the measurements. Using the overall ICC (0.72), hypothetical true risk estimates would be attenuated by ca. $11 \%\left(\mathrm{RR}_{\text {true }}=1.5\right), 23 \%$ $\left(\mathrm{RR}_{\text {true }}=2.5\right)$ and $30 \%\left(\mathrm{RR}_{\text {true }}=3.5\right)$ due to intra-individual chemerin variability. Sensitivity analyses showed a slight improvement of the ICC $(0.77(0.71,0.82))$ when participants with extreme (within 1st or above 99th percentile) chemerin concentrations were excluded. The ICC did not change when non-fasted participants were excluded, while in non-fasted participants the ICC was slightly reduced $(0.70(0.48,0.84))$. Linear regression analyses of chemerin concentrations according to time of day showed no effect at both sampling time points (beta coefficients corresponding to change of chemerin concentration $(\mathrm{ng} / \mathrm{mL})$ per hour of day $(95 \% \mathrm{CI}): 1$ st: 0.73 (-5.94; 7.40), 2nd: $2.69(-4.41 ; 9.78)$ ).

We observed higher chemerin concentrations in obese participants as compared to non-obese (Table 2). Chemerin concentrations were also positively correlated with BMI (rho=0.35 (95\%-CI: 0.22, 0.47)) and waist
() 2017 The authors Published by Bioscientifica Ltd

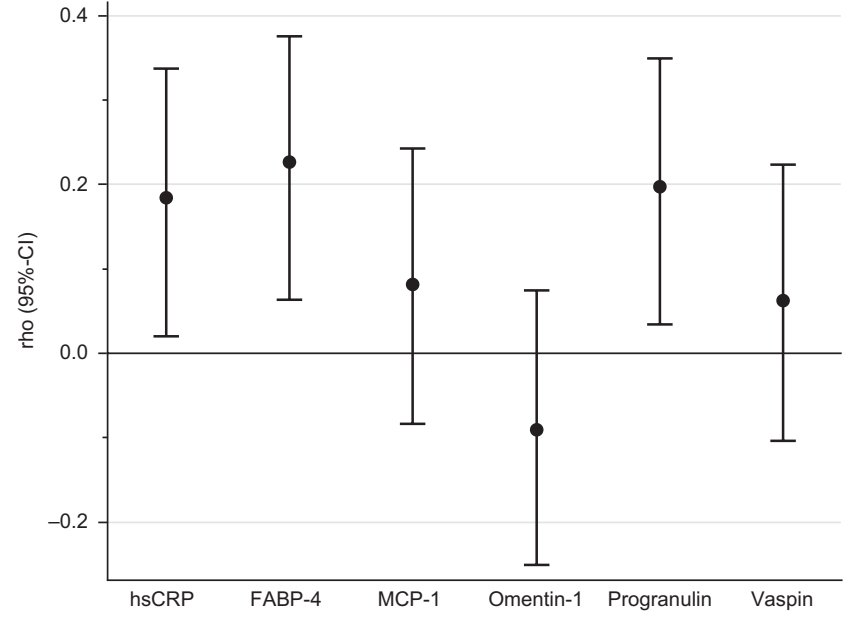

Figure 2

Spearman correlation coefficients $(95 \% \mathrm{Cl})$ for chemerin with inflammation-related biomarkers adjusted for age, sex, BMI and mutually with remaining biomarkers. Analysis based on $n=151$ due to insufficient serum volume for hsCRP measurements. $95 \% \mathrm{Cl}$ calculated using Fisher's $z$ transformation. FABP-4, fatty acid-binding protein-4; hsCRP, high-sensitivity C-reactive protein; MCP-1, monocyte chemoattractant protein 1.

circumference $(0.37(0.24,0.48))$. However, when adjusting for hsCRP and adipokines the correlations were greatly attenuated (BMI: $0.12(-0.05,0.28)$, waist circumference: $0.16(0.00,0.32))$. Chemerin showed a correlation with hsCRP $(0.26(0.10,0.41))$, as well as with the adipokines FABP-4 $(0.28(0.12,0.42))$ and progranulin (0.23 (0.07, $0.38)$ ), when adjusted for age, sex and BMI. No correlation

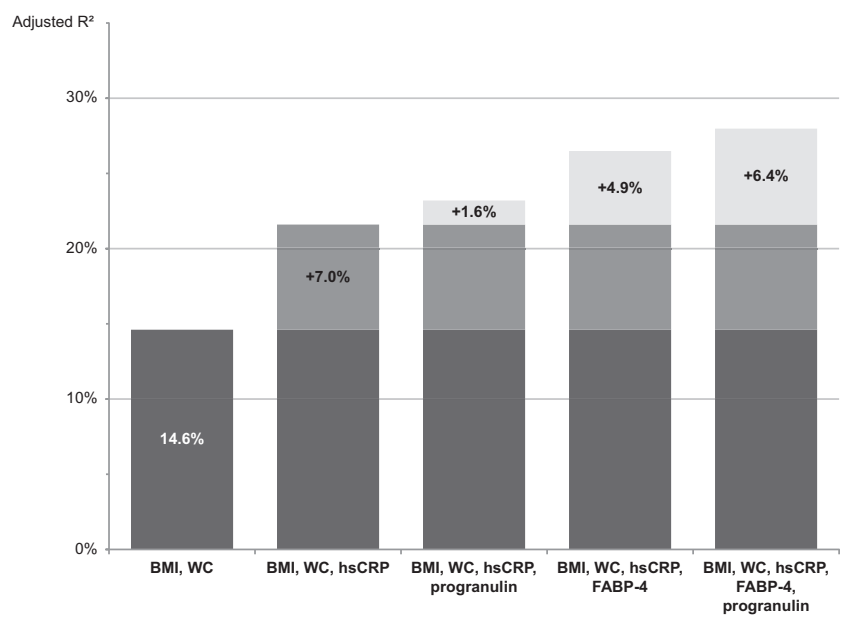

Figure 3

Percentages of explained variance (represented by adjusted $R^{2}$ ) of chemerin concentrations. Adjusted $R^{2}$ was derived from linear regression models with chemerin concentrations as dependent variable and anthropometric measures and biomarker concentrations as independent variables. Models are based on data of participants with available hsCRP measurements $(n=151)$. Adjusted $R^{2}$, adjusted coefficient of determination; FABP-4, fatty acid-binding protein-4; hsCRP, highsensitivity C-reactive protein; WC, waist circumference. http://www.endocrineconnections.org DOI: 10.1530/EC-17-0098 
was observed with MCP-1, omentin-1 and vaspin. Mutual adjustments did not essentially change the correlations of the biomarkers with chemerin (Fig. 2). Linear regression analyses showed that the addition of hsCRP to a model including BMI and waist circumference (adjusted $R^{2}=14.6 \%$ ) increased explained variance of chemerin concentrations by 7.0 percentage points (Fig. 3). Additionally including progranulin or FABP-4 produced even further increase in explained variance by 1.6 and 4.9 percentage points indicating FABP- 4 and progranulin as independent predictors of chemerin concentrations. A model comprising all mentioned variables (BMI, waist circumference, hsCRP, progranulin and FABP-4) resulted in $28.0 \%$ explained variance.

\section{Discussion}

Here, we provide first results for the reliability of chemerin. Our data suggest that chemerin measurements have a good reproducibility over a 4-month time period indicating that single chemerin measurements in prospective studies would be suitable and informative for research. In addition, we report for the first time on interrelations between chemerin and inflammation-related adipokines. Taken together, the data support further prospective studies aimed at improving our understanding of chemerin actions in humans and its potential role in the pathogenesis of inflammation-related diseases and conditions.

Chemerin has been proposed to play a role as adipokine (21), chemokine (22) and a growth factor (23). Although the different pathways are intertwined, chemerin's implication in inflammatory processes seems particularly attractive. Inflammation-related adipokines in general have been suggested to pose a link between excess fat mass, adipose tissue inflammation and chronic diseases and chemerin could therefore play a prominent role in this regard. In line with previous research, we observed higher chemerin concentrations in obese participants as compared to non-obese (24). Expectedly, chemerin concentrations were also positively correlated with BMI and WC independent of participants' age and sex.

Correlation of circulating chemerin levels with markers of low-grade chronic inflammation, such as CRP (24), further hint at chemerin's involvement in inflammatory pathways. In addition to CRP and going beyond what has been shown in humans so far, we showed positive associations with the inflammation-related adipokines FABP-4 and progranulin. Similar to chemerin, these biomarkers were shown to exhibit elevated circulating

http://www.endocrineconnections.org DOI: 10.1530/EC-17-0098
(๔) 2017 The authors Published by Bioscientifica Ltd concentrations in obesity, but also to represent activated immune response due to infection and inflammation $(25,26)$ and act in a pro-inflammatory fashion $(27,28)$. Importantly, FABP-4 and progranulin were independent predictors of chemerin concentrations even beyond BMI, WC and CRP. These data potentially uncover an important interrelation between inflammation-related adipokines that deserve further exploration and careful interpretation. Revealed correlations both with obesity and inflammatory biomarkers essentially add to the growing body of evidence implicating chemerin as a biomarker linking immunity and metabolism in relation to chronic disease risk and point to pathways implicated in adipokine production beyond adiposity per se.

Our study has several strengths. The study population was reasonably large for a validation sample and included both sexes in acceptable proportions. However, the used study population does not allow an unconditional generalization of our results due to specific ethnicity, age range and apparent healthiness of the participants. Future studies should therefore take into account any differences in assays, laboratories, sample types, storage time, repeated samplings and timing of blood draw. However, regarding the last point, timing of blood draw in this study was preferably conducted in the morning, with only few exceptions. In addition, we explored the potential effect of time of the day at blood draw and found no influence.

In summary, we found chemerin to be a reliable biomarker potentially linking immunity and metabolism. This supports the conduct of prospective studies to further the understanding of chemerin's role in the development of chronic diseases.

Declaration of interest

The authors declare that there is no conflict of interest that could be perceived as prejudicing the impartiality of the research reported.

\section{Funding}

The recruitment phase of the EPIC-Potsdam Study was supported by the Federal Ministry of Science of Germany (01 EA 9401) and the European Union (SOC 95201408 05F02). The follow-up was supported by German Cancer Aid (70-2488-Ha I) and the European Community (SOC 98200769 05F02). K Aleksandrova has been awarded a grant by the German Research Foundation (DFG; grant AL 1784/3-1) and was supported by internal funding of the German Institute of Human Nutrition Potsdam-Rehbruecke. The publication of this article was funded by the Open Access Fund of the Leibniz Association.

\section{Author contribution statement}

F E analyzed and interpreted the data and wrote the manuscript; C W contributed in setting up the study and critically revised the manuscript. $R$ G assisted in data analysis and critical revision of the manuscript. R B and 
B I performed the chemerin measurements and quality control procedures; M S critically revised the manuscript; $H$ B designed and conducted the reliability study and critically revised the manuscript; $K$ A conceived the study, planned and supervised data analysis, interpreted data and wrote the manuscript.

\section{Acknowledgements}

The authors thank the Human Study Center at the DIfE for data collection and biological sample logistics. They express thanks to $\mathrm{Dr}$ Manuela Bergmann for her contribution by leading the underlying processes of data generation, as well as to Silke Navia Fruth and Herbert Piechot for their valuable assistance with biosample management. Particular thanks are given to the study data manager Ellen Kohlsdorf. They also express special thanks to Katrin Sprengel and Tanja Ahrens (Department of Clinical Nutrition, DIfE) for their valuable work on measurements of inflammatory adipokines included in the correlation analyses. Finally, we express our gratitude to EPIC-Potsdam participants for their invaluable contribution to the study.

\section{References}

1 Cash JL, Hart R, Russ A, Dixon JP, Colledge WH, Doran J, Hendrick AG, Carlton MB \& Greaves DR. Synthetic chemerin-derived peptides suppress inflammation through ChemR23. Journal of Experimental Medicine 2008205 767-775. (doi:10.1084/jem.20071601)

2 Bozaoglu K, Bolton K, McMillan J, Zimmet P, Jowett J, Collier G, Walder K \& Segal D. Chemerin is a novel adipokine associated with obesity and metabolic syndrome. Endocrinology 2007148 4687-4694. (doi:10.1210/en.2007-0175)

3 Goralski KB, McCarthy TC, Hanniman EA, Zabel BA, Butcher EC, Parlee SD, Muruganandan S \& Sinal CJ. Chemerin, a novel adipokine that regulates adipogenesis and adipocyte metabolism. Journal of Biological Chemistry 2007282 28175-28188. (doi:10.1074/ jbc.M700793200)

4 Nagpal S, Patel S, Jacobe H, DiSepio D, Ghosn C, Malhotra M, Teng M, Duvic M \& Chandraratna RAS. Tazarotene-induced Gene 2 (TIG2), a novel retinoid-responsive gene in skin. Journal of Investigative Dermatology 1997109 91-95. (doi:10.1111/1523-1747. ep12276660)

$5 \mathrm{Hu}$ W \& Feng P. Elevated serum chemerin concentrations are associated with renal dysfunction in type 2 diabetic patients. Diabetes Research and Clinical Practice 201191 159-163. (doi:10.1016/ j.diabres.2010.11.016)

6 Kaur J, Adya R, Tan BK, Chen J \& Randeva HS. Identification of chemerin receptor (ChemR23) in human endothelial cells: chemerininduced endothelial angiogenesis. Biochemical and Biophysical Research Communications 2010391 1762-1768. (doi:10.1016/ j.bbrc.2009.12.150)

7 Takahashi M, Takahashi Y, Takahashi K, Zolotaryov FN, Hong KS, Kitazawa R, Iida K, Okimura Y, Kaji H, Kitazawa S, et al. Chemerin enhances insulin signaling and potentiates insulin-stimulated glucose uptake in 3T3-L1 adipocytes. FEBS Letters 2008582 573-578. (doi:10.1016/j.febslet.2008.01.023)

8 Ferland DJ \& Watts SW. Chemerin: a comprehensive review elucidating the need for cardiovascular research. Pharmacological Research 201599 351-361. (doi:10.1016/j.phrs.2015.07.018)

9 Weigert J, Obermeier F, Neumeier M, Wanninger J, Filarsky M, Bauer S, Aslanidis C, Rogler G, Ott C, Schaffler A, et al. Circulating levels of chemerin and adiponectin are higher in ulcerative colitis and chemerin is elevated in Crohn's disease. Inflammatory Bowel Diseases 201016 630-637. (doi:10.1002/ibd.21091)

10 Yilmaz Y, Yonal O, Kurt R, Alahdab YO, Eren F, Ozdogan O, Celikel CA, Imeryuz N, Kalayci C \& Avsar E. Serum levels of omentin, chemerin and adipsin in patients with biopsy-proven nonalcoholic fatty liver disease. Scandinavian Journal of Gastroenterology 201146 91-97. (doi:10.3109/00365521.2010.516452)

11 Adrych K, Stojek M, Smoczynski M, Sledzinski T, Sylwia SW \& Swierczynski J. Increased serum chemerin concentration in patients with chronic pancreatitis. Digestive and Liver Disease 201244 393-397. (doi:10.1016/j.dld.2011.06.020)

12 Tan BK, Chen J, Farhatullah S, Adya R, Kaur J, Heutling D, Lewandowski KC, O'Hare JP, Lehnert H \& Randeva HS. Insulin and metformin regulate circulating and adipose tissue chemerin. Diabetes 200958 1971-1977. (doi:10.2337/db08-1528)

13 Li Y, Shi B \& Li S. Association between serum chemerin concentrations and clinical indices in obesity or metabolic syndrome: a meta-analysis. PLOS ONE 20149 e113915. (doi:10.1371/journal. pone.0113915)

14 Gallo V, Egger M, McCormack V, Farmer PB, Ioannidis JPA, Kirsch-Volders M, Matullo G, Phillips DH, Schoket B, Stromberg $\mathrm{U}$, et al. STrengthening the Reporting of OBservational studies in Epidemiology-Molecular Epidemiology (STROBE-ME): an extension of the STROBE statement. PLoS Medicine 20118 e1001117. (doi:10.1371/ journal.pmed.1001117)

15 Riboli E, Hunt KJ, Slimani N, Ferrari P, Norat T, Fahey M, Charrondiere UR, Hemon B, Casagrande C, Vignat J, et al. European Prospective Investigation into Cancer and Nutrition (EPIC): study populations and data collection. Public Health Nutrition 20025 1113-1124. (doi:10.1079/PHN2002394)

16 Boeing H, Wahrendorf J \& Becker N. EPIC-Germany - a source for studies into diet and risk of chronic diseases. European investigation into cancer and nutrition. Annals of Nutrition and Metabolism 199943 195-204. (doi:10.1159/000012786)

17 Peters T, Brage S, Westgate K, Franks PW, Gradmark A, Diaz MJT, Huerta JM, Bendinelli B, Vigl M, Boeing H, et al. Validity of a short questionnaire to assess physical activity in 10 European countries. European Journal of Epidemiology 201227 15-25. (doi:10.1007/s10654011-9625-y)

18 Eichelmann F, Rudovich N, Pfeiffer AF, Schulze MB, di Giuseppe R, Boeing $\mathrm{H}$ \& Aleksandrova K. Novel adipokines: methodological utility in human obesity research. International Journal of Obesity $2017 \mathbf{4 1}$ 976-998. (doi:10.1038/ijo.2017.68)

19 Wittenbecher C, di Giuseppe R, Biemann R, Menzel J, Arregui M, Hoffmann J, Aleksandrova K, Boeing H, Isermann B, Schulze $\mathrm{MB}$, et al. Reproducibility of retinol binding protein 4 and omentin-1 measurements over a four months period: a reliability study in a cohort of 207 apparently healthy participants. PLOS ONE 201510 e0138480. (doi:10.1371/journal.pone.0138480)

20 Bland JM \& Altman DG. Measuring agreement in method comparison studies. Statistical Methods in Medical Research 19998 135-160. (doi:10.1191/096228099673819272)

21 Roh SG, Song SH, Choi KC, Katoh K, Wittamer V, Parmentier M \& Sasaki S. Chemerin - a new adipokine that modulates adipogenesis via its own receptor. Biochemical and Biophysical Research Communications 2007362 1013-1018. (doi:10.1016/j.bbrc.2007.08.104)

22 Wittamer V, Franssen JD, Vulcano M, Mirjolet JF, Le Poul E, Migeotte I, Brezillon S, Tyldesley R, Blanpain C, Detheux M, et al. Specific recruitment of antigen-presenting cells by chemerin, a novel processed ligand from human inflammatory fluids. Journal of Experimental Medicine 2003198 977-985. (doi:10.1084/ jem.20030382)

23 Bozaoglu K, Curran JE, Stocker CJ, Zaibi MS, Segal D, Konstantopoulos N, Morrison S, Carless M, Dyer TD, Cole SA, et al. Chemerin, a novel adipokine in the regulation of angiogenesis. Journal of Clinical Endocrinology and Metabolism 201095 2476-2485. (doi:10.1210/ jc.2010-0042)

24 Zylla S, Pietzner M, Kuhn JP, Volzke H, Dorr M, Nauck M \& Friedrich N. Serum chemerin is associated with inflammatory and metabolic parameters-results of a population-based study. Obesity 201725 468-475. (doi:10.1002/oby.21735)

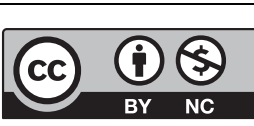

This work is licensed under a Creative Commons Attribution-NonCommercial 4.0 International License. 
25 Hotamisligil GS \& Bernlohr DA. Metabolic functions of FABPsmechanisms and therapeutic implications. Nature Reviews Endocrinology 201511 592-605. (doi:10.1038/nrendo.2015.122)

26 Jian JL, Konopka J \& Liu CJ. Insights into the role of progranulin in immunity, infection, and inflammation. Journal of Leukocyte Biology 201393 199-208. (doi:10.1189/jlb.0812429)

27 Hui X, Li H, Zhou Z, Lam KS, Xiao Y, Wu D, Ding K, Wang Y, Vanhoutte PM \& Xu A. Adipocyte fatty acid-binding protein modulates inflammatory responses in macrophages through a positive feedback loop involving c-Jun NH2-terminal kinases and activator protein-1. Journal of Biological Chemistry 2010285 10273-10280. (doi:10.1074/jbc.M109.097907)

28 Youn BS, Bang SI, Kloting N, Park JW, Lee N, Oh JE, Pi KB, Lee TH, Ruschke K, Fasshauer M, et al. Serum progranulin concentrations may be associated with macrophage infiltration into omental adipose tissue. Diabetes 200958 627-636. (doi:10.2337/db08-1147)

Received in final form 1 June 2017

Accepted 6 June 2017

Accepted Preprint published online 7 June 2017
This work is licensed under a Creative Commons Attribution-NonCommercial 4.0 International License. 